\title{
Methylene blue as an early diagnostic marker for oral precancer and cancer
}

\author{
Akhtar Riaz, Balasundari Shreedhar, Mala Kamboj* and S Natarajan
}

\begin{abstract}
Oral cancer is one of the most common neoplasm's and is ranked eighth in the cancer incidence worldwide. Early detection is of critical importance because survival rates markedly improve. In vivo staining is a simple, inexpensive, and fairly sensitive method. Involved 120 patients (50 with Premalignant Lesion, 50 with OSCC and 20 controls) stained by Methylene Blue (MB). The results of MB uptake were compared with a simultaneous biopsy of these lesions. Pathologically confirmed precancers and cancers were the positive targets of this screening, while hyperkeratosis without dysplasia and no evidence of malignancy were sorted as negative subjects of screening. The results revealed sensitivity of $91.4 \%$, specificity of $66.6 \%$, positive predictive value $97.7 \%$ and negative predictive value $33 \%$ leading to diagnostic accuracy of MB stain to $90 \%$. We state that MB staining is useful diagnostic tool in community oral cancer screening programmes for high-risk individuals.
\end{abstract}

Keywords: Methylene blue, Oral precancer, Oral cancer, Early marker

\section{Introduction}

Oral cavity cancer accounts for approximately 3\% of all malignancies and is a significant worldwide health problem (Kademani 2007; Silverman 2001). Most oral malignancies occur as squamous cell carcinomas; despite remarkable advances in treatment modalities, the 5-year survival rate has not significantly improved over the past several decades and still hovers at about $50-60 \%$ (Ries et al. 2999). Oral squamous cell carcinoma (OSCC) is one of the most common neoplasm and it is ranked eighth in the cancer incidence worldwide, with an annual incidence rate of 64,460 . However total number of cases at any given time will be 2.5 to 3 times higher than this number (Mehta \& Hamner 1993).

Many OSCCs develop from premalignant lesions \& conditions of the oral cavity (Silverman et al. 1984; Silverman 1968). A wide array of conditions have been implicated in the development of oral cancer, including leukoplakia, erythroplakia, palatal lesion of reverse cigar smoking, oral submucous fibrosis, discoid lupus erythematosus, and hereditary disorders such as dyskeratosis congenita and epidermolysis bullosa (Warnakulasuriya

\footnotetext{
* Correspondence: malskam@gmail.com

Department of Oral Pathology and Microbiology, Career Post Graduate Institute of Dental Sciences and Hospital, C-1111, Indiranagar, Lucknow 226016, India
}

et al. 2007). Despite the general accessibility of the oral cavity during physical examination, many malignancies are not diagnosed until late stages of disease. In order to prevent malignant transformation of these precursor lesions, multiple screening and detection techniques have been developed to address this problem. The early detection of cancer is of critical importance because survival rates markedly improve when the oral lesion is identified at an early stage (Ries et al. 2999). Periodic clinical examination of the oral cavity is the key to early detection of oral cancer (Ya-Wei 2007a). Among the diagnostic tool, in vivo staining is advocated as a simple, inexpensive and fairly sensitive method (Ya-Wei 2007b).

Methylene Blue (MB) has been used to detect gastric, prostrate and bladder cancers (Mufti et al. 1990; Gill et al. 1984; Yu et al. 1990). In the diagnosis, accuracy of the $\mathrm{MB}$ technique is used for identification of intestinal metaplasia, carcinoma or dysplasia. In one of the studies, its validity in detection of oral cancer and precancerous lesion has also been examined. The exact mechanism for the uptake of methylene blue in epithelial tissue may resemble that of toluidine blue in the acidophilic characteristic of cells with abnormal concentration of nucleic acid, resulting in differential uptake between normal/ benign and highly dysplastic /malignant cells. The 90\% sensitivity of MB was no less than $72-100 \%$ sensitive

\section{Springer}

(c) 2013 Riaz et al.; licensee Springer. This is an Open Access article distributed under the terms of the Creative Commons Attribution License (http://creativecommons.org/licenses/by/2.0), which permits unrestricted use, distribution, and reproduction in any medium, provided the original work is properly cited. 
report with toluidine blue staining. Considering the low toxicity and cheaper prices, methylene blue may be conveniently substituted for large scale screening in high risk patients (Ya-Wei 2007a). Methylene blue is a commonly used stain that helps us see microscopic life in brilliant color. The dye, methylene blue shows the deepest shade of blue, indicating a strong attraction to acids including DNA.

In the present paper an attempt has been made to establish $\mathrm{MB}$ as an early diagnostic marker in the suspected cases of oral precancerous lesions and oral squamous cell carcinoma for wider screening programmes.

\section{Methods}

\section{Subjects}

The study group consisted of 120 subjects of both the sexes, 50 subjects with clinically suspicious premalignant lesions, 50 subjects with clinically suspicious oral cancers and 20 cases of subjects with clinically appearing normal mucosa. A provisional diagnosis of leukoplakia, erythroplakia, smoker's palate (premalignant lesions) and Oral cancer were made on basis of clinical examination. Patient's more than or equal to 15 yrs of age, with the habit of smoking and/or tobacco consumption were made part of the study. Patients with age less than 15 years, immunocompromised states, with premalignant conditions, and patients with diabetes or other systemic disorders were excluded from the study. Ethics committee approval from the college ethics committee was taken for the smooth conduct of the study. (CPGIDSH/09). Controls were subjects with clinically normal appearing oral mucosa. The subjects were made to sit comfortably on the dental chair and were thoroughly examined under artificial illumination. The clinical examination was done and the relevant data was entered into the proforma.

\section{Gargling solution}

Methylene blue dye system had 2 solution bottles. The dye rinse solution (Bottle A) had 1\% methylene blue, 1\% malachite, $0.5 \%$ eosin, glycerol, and dimethylsulfoxide. Pre- and post-rinse solution (Bottle B) had 1\% lactic acid, and purified water.

\section{Staining procedure}

The application of methylene blue was as follows. The patients were directed to rinse their mouth with $1 \%$ lactic acid \& distilled water for 30 seconds to remove food debris and excess saliva and to provide a consistent oral environment. The mucosa of the target area was gently dried with gauze and power air spray to ensure that the lesion was not being contaminated with saliva. The dye was directly applied on the lesion with help of cotton bud (Mufti et al. 1990) first and after used as a mouth rinse gargle (Ya-Wei 2007a) with Methylene blue for
30 seconds; then expectorated. Patients then rinsed again with $1 \%$ lactic acid for 30 seconds to wash out the excess dye (Ya-Wei 2007a). The pattern of dye retention was assessed by the intensity of stain retention on the lesion. Local, and deep blue stains were marked as positive (+) reaction. Wide, shallow or faint blue stains were marked as negative (-) reaction (Ya-Wei 2007a). The results of methylene blue dye staining were recorded with photographs and incisional biopsy was performed simultaneously in the suspected lesions to compare the accuracy of the diagnostic capability of methylene blue.

\section{Biopsy}

Incisional biopsy was performed in the most obvious staining area of the suspected lesion of the patient under local anaesthesia. The specimen was then fixed in $10 \%$ neutral buffered formalin and processed in the oral pathology laboratory for initial routine pathologic diagnosis.

\section{Histological examination}

All the specimens were microscopically evaluated by pathologists who were blind to the results of methylene blue stain. The pathology reports of the lesions were classified as A) Oral Precancerous: Mild, Moderate and Severe Dysplasia B) Oral Cancer: Well, Moderately and Poorly differentiated Squamous Cell Carcinoma C) Lesions neither precancerous nor malignant: Hyperkeratosis without dysplasia and No evidence of malignancy. The results were then deduced and were statistically analyzed using the various statistical tests given below and the final results were drawn.

\section{Statistical analysis}

The pathologically proven OSCC and precancerous lesions were the targets of screening and the concerned is presented by number and percentage of the same. The results of positive/negative uptake of methylene blue in each lesion were correlated with the histopathological diagnosis. Statistical analysis was performed, including sensitivity, specificity, positive and negative predictive values. The association of methylene blue uptake and pathologic diagnosis among the precancers/ OSCC group, lesions neither premalignant nor malignant group, nor normal group were analyzed using Fisher's exact test (Wilson 1927). A p value (probability value) of less than 0.01 was considered very significant. The statistical analysis was done using Critical Appraisal Diagnostic Test and GraphPad Software.

\section{Results}

\section{Subject characteristics}

120 subjects (50 with Premalignant Lesion, 50 with OSCC and 20 controls) were enrolled in this study. The patient's ages (patient group) ranged from 15 yrs to 80 yrs, with the 
ratio of male to female being $5: 1$. Both the groups gave history of tobacco consumption. The suspected lesions were distributed over the buccal mucosa $(n=84)$, palate $(n=6)$, tongue $(n=4)$, floor of the mouth $(n=2)$. In the control group, as methylene blue dye was not used to examine the oral cavity, it was necessary to verify that the dye would not be retained on normal mucosa. The results demonstrated that there was no retained dye in the control group.

\section{Methylene blue staining related to grade of pathology}

The Clinical and Histological characteristics of the various study Groups are shown in Table 1. The pathologic grade was classified as precancer lesions, oral cancer lesions, hyperkeratosis without dysplasia and no evidence of malignancy. The following statistical terms were used to describe and analyze the relationship between the grade of pathology and the uptake of methylene blue staining. Sensitivity represents the proportion of histologically proved cancer/precancerous lesions which are detected by positive methylene blue staining. In the current study, 44 of 48 pathologically proven cancers and $42 / 46$ precancerous lesions were positive with deep and focal methylene blue staining (Figure 1A,B,C, Figure $2 \mathrm{~A}, \mathrm{~B}, \mathrm{C})$. The overall sensitivity was $91.4 \%$.

Specificity suggests the proportion of pathologic benign lesions, neither precancerous lesions nor cancers, which are correctly identified as negative staining of methylene blue. In our study, 4 out of 6 cases with no dysplasia or malignancy showed negative staining; thus, the specificity was $66.6 \%$. The results of staining with methylene blue for all lesions correlated well with the pathologic diagnosis and are summarized in Table 2. Fisher's exact test showed significant differences among cancer/precancerous lesions, lesions without dysplasia or malignancy, and normal control groups $(\mathrm{p}<0.001)$. Overall, the positive predictive value was $97.7 \%$, and the false predictive value was $33.3 \%$.

\section{Overall diagnostic validity of methylene blue stain}

Out of 100 lesions, 88 (92\%) lesions retained methylene blue stain while $12(8 \%)$ lesions failed to retain the stain. Out of these 88 methylene blue stained lesions, 86 were histologically proved as having either dysplastic or carcinomatous change, while $2(2 \%)$ were diagnosed as hyperkeratosis without dysplasia / no evidence of malignancy. Out of 12 unstained lesions 8 were histopathologically diagnosed as carcinomatous while 4 were diagnosed as hyperkeratosis without dysplasia / no evidence of malignancy respectively. The sensitivity of methylene blue in determining dysplastic and carcinomatous changes was determined as $91.4 \%$ while the specificity was determined as $66.6 \%$, the positive predictive value $97.7 \%$, the negative predictive value $33.3 \%$. The overall diagnostic value of methylene blue stain in distinguishing premalignant and malignant lesion 90\% The $\mathrm{p}$ value less than $<0.001$ which is indicates that these results are stastically very significant.

\section{Discussion}

Oral Cancer is very common in a country like India due to habits of smoking and chewing tobacco containing pan masala. Tobacco consumption is the main etiologic factor inducing carcinogenesis in oral mucosa. WHO

Table 1 Clinical and histological characteristics of the various study groups

\begin{tabular}{|c|c|c|c|}
\hline \multirow[t]{2}{*}{ Criteria } & Group I & Group II & Group III \\
\hline & Premalignant lesions $(n=50)$ & $\operatorname{OsCC}(n=50)$ & Controls $(n=20)$ \\
\hline Age $\leq 40$ yrs & $2856 \%$ & $816 \%$ & $1260 \%$ \\
\hline$\geq 40$ yrs & $2244 \%$ & $4284 \%$ & $840 \%$ \\
\hline Sex Females & $24 \%$ & $1428 \%$ & $420 \%$ \\
\hline Males & $4896 \%$ & $3672 \%$ & $1680 \%$ \\
\hline Site Buccal Mucosa(BM) & 40 leukoplakia & $44 \mathrm{BM}$ with vest. ridge & - - \\
\hline Palate & 4 smoker's palate & 2 & \\
\hline Tongue & 2 leukoplakia & 2 & \\
\hline Floor of mouth & & 2 & \\
\hline Tobacco Use No & 0 & 0 & 20 \\
\hline Yes & 50 & 50 & 0 \\
\hline \multirow[t]{5}{*}{ Histological Diagnosis } & Mild 10 20\% & WDSCC 30 60\% & - \\
\hline & Moderate 34 68\% & MDSCC 16 32\% & \\
\hline & Severe $24 \%$ & PDSCC $24 \%$ & \\
\hline & Hyperkeratosis 4 8\% & No evidence of $24 \%$ & \\
\hline & without dysplasia & Malignancy & \\
\hline
\end{tabular}




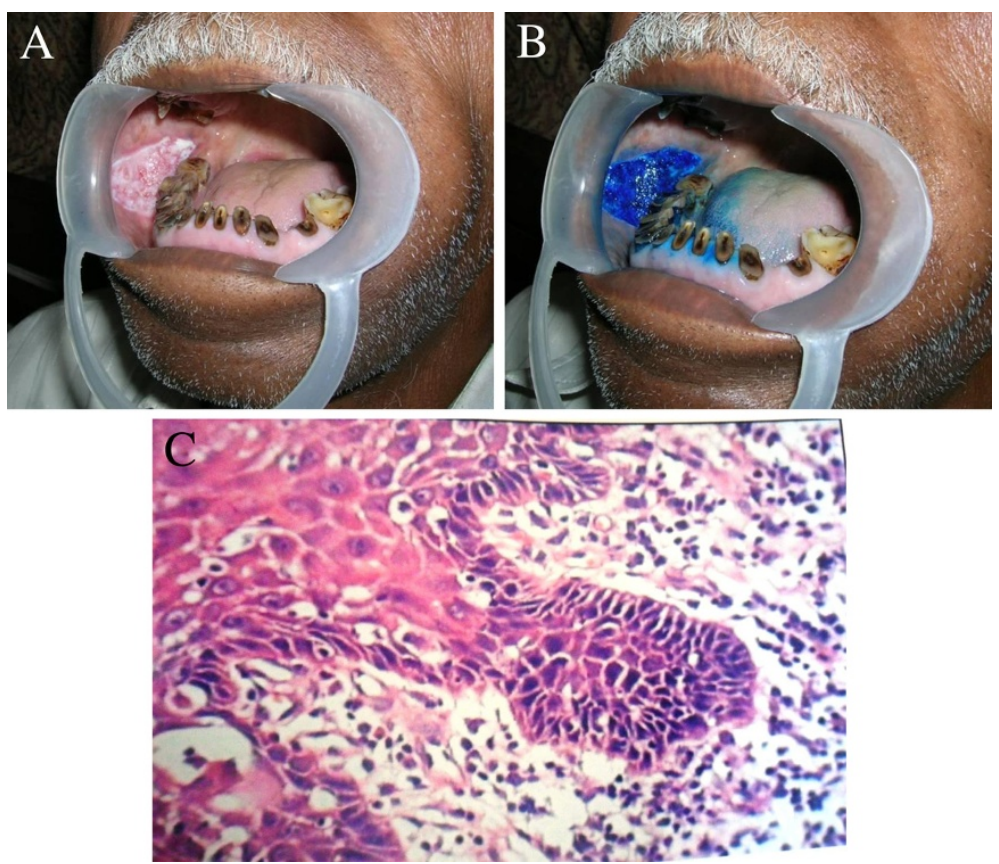

Figure 1 Presentation of a true-positive staining on a red and white homogenous patch on the right buccal mucosa. (A) The lesion presented clinically as a red and white homogenous patch. (B) Vital staining with methylene blue showed deep and focal staining of the lesion. (C) The final pathology revealed a severe dysplasia. (H \&E, 40X).
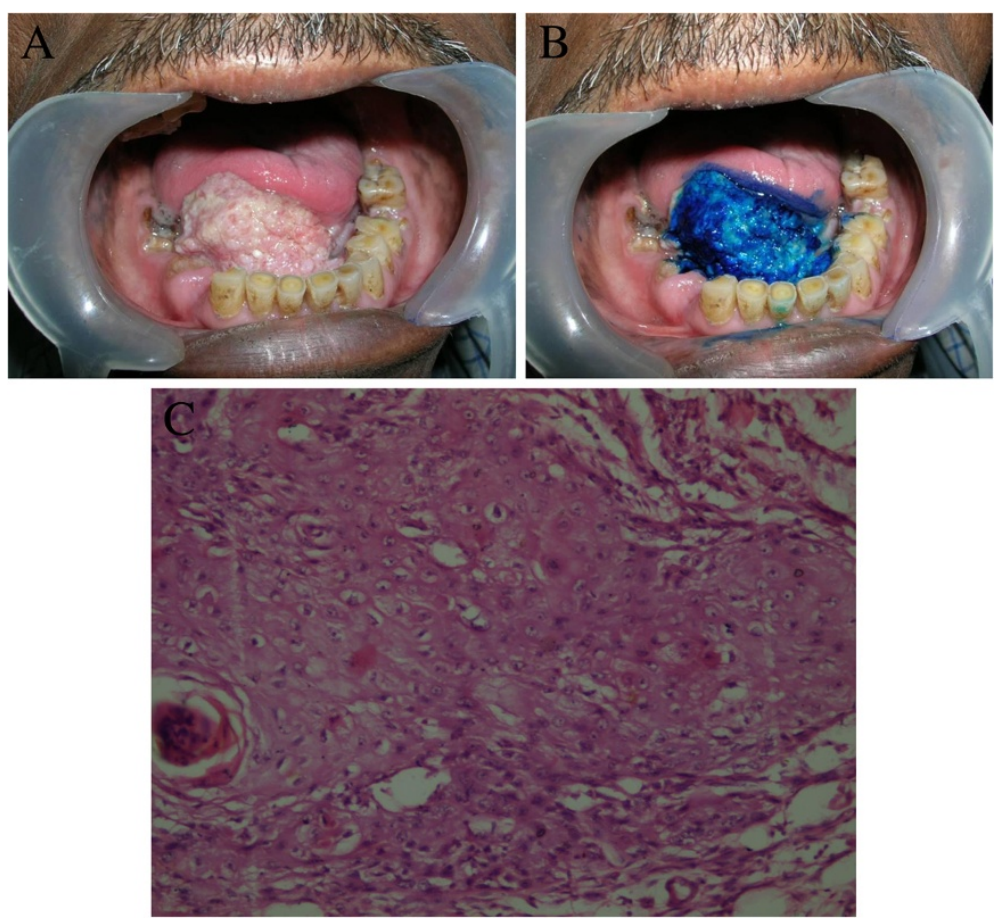

Figure 2 Presentation of a true-positive staining on the floor of the mouth. (A) The lesion presented clinically as a huge growth on the floor of the mouth with indurated margins. (B) Vital staining with methylene blue showed deep and focal staining of the lesion. (C) The final pathology revealed a moderately differentiated squamous cell carcinoma. (H \&E, 40X). 
Table 2 Diagnostic accuracy of methylene blue in the study groups

\begin{tabular}{|c|c|c|c|c|c|}
\hline \multirow{3}{*}{$\begin{array}{l}\text { Methylene Blue } \\
\text { Retention }\end{array}$} & \multicolumn{4}{|c|}{ Histological diagnosis } & \multirow[t]{3}{*}{ Overall (PML + SCC) } \\
\hline & \multicolumn{2}{|c|}{ Premalignant Lesion (PML) } & \multicolumn{2}{|c|}{ Squamous Cell Carcinoma (SCC) } & \\
\hline & Dysplasia & $\begin{array}{c}\text { Hyperkeratosis } \\
\text { without dysplasia }\end{array}$ & oscc & $\begin{array}{l}\text { No evidence } \\
\text { of malignancy }\end{array}$ & \\
\hline+ & 42 & 1 & 44 & 1 & \\
\hline- & 4 & 3 & 4 & 1 & \\
\hline Sensitivity & \multicolumn{2}{|r|}{$91.3 \%$} & \multicolumn{2}{|r|}{$91.6 \%$} & $91.4 \%$ \\
\hline Specificity & \multicolumn{2}{|r|}{$75 \%$} & \multicolumn{2}{|r|}{$50 \%$} & $66.6 \%$ \\
\hline Positive predictive value & \multicolumn{2}{|r|}{$97.6 \%$} & \multicolumn{2}{|r|}{$97.7 \%$} & $97.7 \%$ \\
\hline Negative Predictive Value & \multicolumn{2}{|r|}{$42.8 \%$} & \multicolumn{2}{|r|}{$20 \%$} & $33.3 \%$ \\
\hline Diagnostic Accuracy & \multicolumn{2}{|r|}{$90 \%$} & \multicolumn{2}{|r|}{$90 \%$} & $90 \% \mathbf{p}<0.0001$ (very significant) \\
\hline
\end{tabular}

indicates a 500 patients increase in cancer by 2025 of which 220 will be due to tobacco use. A survey by WHOSEA (Women's Health in South East Asia) has indicated that almost $50 \%$ of oral cancer in men and $25 \%$ cases of women in India are believed to be tobaccoborne (Mehta \& Hamner 1993). Thus oral screening of high-risk individuals is very important in these countries. Amongst the various diagnostic tools, in vivo staining is advocated as simple and sensitive method for early detection of oral cancer (Ya-Wei 2007b).

For a large scale community screening, some dye materials easily help to identify abnormal mucosa tissue which raise oral examiners attention and refer the patients with suspicious lesions to oral surgeons for further examination. Toluidine Blue has been championed in many parts of world for several decades as a means of identifying clinically occult lesions in patients whose oral mucosa may otherwise be normal that is as a screening test or adjunct (Mashberg 1983). The efficacy of this technique has been evaluated in many reports with diverse results. It has yielded sensitivities between $72-100 \%$ and specificities between $45-67 \%$ in detecting suspicious malignancies (Epstein et al. 1997; Onofre et al. 2001; Epstein et al. 1992; Warnakulasuriya \& Johnson 1996). However the material data safety sheet indicates that toluidine blue is probably toxic by ingestion and is seldom used in detecting cancers in other parts of body (Ya-Wei 2007a).

Methylene blue is another recently proposed dye for in vivo staining used in endoscopic examination (Bruno 2003). Its application has been reported recently in detecting some gastrointestinal abnormalities such as Barrett's esophagus (Canto et al. 1996; Canto et al. 2000), gastric cancer (Canto et al. 2000), prostate cancer (Mufti et al. 1990; Gill et al. 1984) bladder Methylene blue (MB) is an acidophilic dye, it binds to the double helical DNA with a high affinity, as deduced from the absorption and fluorescence spectral data. Extensive hypochromism and red shifts in the absorption spectra were observed when $\mathrm{MB}$ binds to calf thymus DNA (CT DNA), which suggested the intercalation mechanism of $\mathrm{MB}$ into DNA bases. Upon binding to DNA, the fluorescence from MB was efficiently quenched by the DNA bases, with no shifts in the emission maximum. The large increases in the polarization upon binding to CT DNA supported the intercalation of MB into the helix (Wen-You et al. 2000). The binding of methylene blue to DNA and chromatin treated in various ways was also examined by equilibrium dialysis. The maximum $\mathrm{r}$ value (moles of bound dye/mole of nucleotide) was 1.0 for DNA, 0.6 for unfixed chromatin, and 0.83 for chromatin fixed in methanol-acetic acid104. All these studies have established the fact that methylene show proportionate binding to DNA nucleotide and this accounts for increase in intensity of stain color with increase in chromatin material in premalignant and malignant cells.

Very few studies have been conducted till now to establish the usage of methylene blue technique in detecting oral precancerous/cancerous lesions8. Screening studies should always be evaluated with respect to their sensitivity, specificity and predictive values (Lingen et al. 2008). Although there is no defined value for the ideal screening test but in general, it is desirable to have both high specificity (few false positives) and high sensitivity (few false negatives) (Lingen et al. 2008). In the present study, the pool of 100 patients (50 cancerous, 50 precancerous) were screened against 20 control (normal subjects) and we found that 88/94 pathologically proven precancers/cancerous lesions showed positive staining with localized and deep blue stain. Overall 91.4\% sensitivity (91.3\% for premalignant and $91.6 \%$ for OSCC) was reported with a false negative rate of $8.6 \%$. Previous studies have indicated sensitivity of $72-100 \%$ (Epstein et al. 1997; Onofre et al. 2001; Epstein et al. 1992; Warnakulasuriya \& Johnson 1996) in others and 90\% in oral screening (Ya-Wei 2007a), on the basis of these and our studies we can say that using methylene blue dye for diagnostic screening is highly acceptable. For few false negatives results, we consider that ambiguous faint blue 
shallow stains, which may be misinterpreted as negatives but clinically suspicious of malignancy, are proved pathologically after biopsy. As far as specificity is concerned we obtained overall specificity of $66.6 \%(4 / 6)$ (75\% in premalignant and $50 \%$ in OSCC) with a resulting 2 false positives, that were the cases of chronic unhealed ulcers. The high false positives rate was discussed to be related to the retention of stain in inflamed and trauma areas (Mashberg 1983). Other factors can be irregular, papillary or digital surfaces of the lesions, which may cause the mechanical retention of dye, contamination of saliva and plaque, retention of dye material in papilla of the tongue or minor salivary gland ducts over the mucosa (Ya-Wei 2007a).

Applying this method for screening high risk patients having habits of tobacco chewing or smoking, a large group of individuals may include those with obvious oral lesions and those with normal oral mucosa. To study these people and to re-evaluate the efficacy of lesions, a large population of the people with normal oral mucosa will lower the rate of false positives and result in higher specificity as found on our case where when compared with control $(n=20)$ false positives were 0 and specificity was $100 \%$ although our control group was having normal mucosa but the flaw in our experimental design was that these subjects were not having any type of tobacco chewing or smoking habits, as we cannot screen individuals with these habits and no lesions because performing biopsy in normal mucosa will be unethical. Thus we can say that this study has established methylene blue as a diagnostic agent (with diagnostic accuracy of $90 \%$ and stastically very significant results with p-value $<0.001$ ) for early detection of cancerous and precancerous lesions. This dye will be convenient to substitute for toluidine blue because of its non-toxicity and low cost. However pathology report based on biopsy will always remain gold standard to exactly and accurately diagnose the lesion before a treatment modality is determined (Mashberg 1980).

Staining should be routinely used as a method to assist the choice of biopsy site and in the follow up of premalignant lesions and in the experienced hands marginal demarcation of the malignant lesions enables an intervention method to be adopted earlier for the diseases, which carries a high rate of morbidity and mortality. Further study is recommended to study the exact binding mechanism of methylene blue in precancerous, cancerous, benign and normal mucosa to establish this dye as the efficient diagnostic tool for diagnosing and differentiating between different pathological conditions.

\section{Competing interest}

The author declares that they have no competing interest.

\section{Authors' contributions}

AR carried out the practical work of the study, BS gave expert comments and provided help in framing the study and the manuscript. MK drafted the manuscript and conceptualized the idea of the study. SN participated in drafting of the manuscript. All authors read and approved the final manuscript.

Received: 3 January 2013 Accepted: 25 February 2013

Published: 9 March 2013

\section{References}

Bruno MJ (2003) Magnification endoscopy, high resolution endoscopy, and chromoscopy; towards a better optical diagnosis. Gut 52(Suppl 4):iv7-iv11, 5

Canto MI, Setrakian S, Petras RE et al (1996) Methylene blue selectively stains intestinal metaplasia in Barrett's esophagus. Gastrointest Endosc 44:1-7

Canto MI, Setrakian S, Willis J et al (2000) Methylene blue-directed biopsies improve detection of intestinal metaplasia and dysplasia in Barrett's esophagus. Gastrointest Endosc 51:560-568

Epstein JB, Scully C, Spinelli J (1992) Toluidine blue and Lugol's iodine application in the assessment of oral malignant disease and lesions at risk of malignancy. J Oral Pathol Med 21:160-163

Epstein JB, Oakley C, Millner A, Emerton S, van der Meij E (1997) The utility of toluidine blue as $\mathrm{s}$ a diagnostic aid in patients previously treated for upper oropharyngeal carcinoma. Oral Surg Oral Med Oral Pathol Oral Radiol Endod 83(5):537-547

Gill WB, Huffman JL, Lyon ES, Bagley DH, Schoenberg HW, Straus FH 2nd (1984) Selective surface staining of bladder tumors by intravesical methylene blue with enhanced endoscopic identification. Cancer 53:2724-2727

Kademani D (2007) Oral cancer. Mayo Clin Proc 82(7):878-887

Lingen MW, Kalmar JR, Karrison T, Speight PM (2008) Critical evaluation of diagnostic aids for the detection of oral cancer. Oral Onc 44:10-22

Mashberg A (1980) Re-evaluation of toluidine blue application as a diagnostic adjunct in the detection of asymptomatic oral squamous carcinoma. Cancer 46:758-763

Mashberg A (1983) Final evaluation of tolonium chloride rinse for screening of highrisk patients with asymptomatic squamous carcinoma. JADA 106:319-323

Mehta FS, Hamner JE (1993) Tobacco related oral mucosal lesions and conditions in India. Tata Inst. of Fundamental, Research

Mufti GR, Shah PJ, Parkinson MC, Riddle PR (1990) Diagnosis of clinically occult bladder cancer by in vivo staining with methylene blue. Br J Urol 65:173-175

Onofre MA, Sposto MR, Navarro CM (2001) Reliability of toluidine blue application in the detection of oral epithelial dysplasia and in situ and invasive squamous cell carcinomas. Oral Surg Oral Med Oral Pat Oral Radiol Endod 91:535-540

Ries LAG, Melbert D, Krapcho M, Stinchcomb DG, Howlader N, Horner MJ, Mariotto A, Miller BA, Feuer EJ, Altekruse SF, Lewis DR, Clegg L, Eisner MP, Reichman M, Edwards BK (2999) SEER cancer statistics review, 1975-2005. National Cancer Institute, Bethesda, MD, http://seer.cancer.gov/csr/1975_ 2005/, based on November 2007 SEER data submission, posted to the SEER web site, 2008 [database online]. 2008. Updated 2008

Silverman S Jr (1968) Observations on the clinical characteristics and natural history of oral leukoplakia. J Am Dent Assoc 76(4):772-777

Silverman S Jr (2001) Demographics and occurrence of oral and pharyngeal cancers. The outcomes, the trends, the challenge. J Am Dent Assoc 132:7S-11S

Silverman S Jr, Gorsky M, Lozada F (1984) Oral leukoplakia and malignant transformation. A follow-up study of 257 patients. Cancer 53(3):563-568

Warnakulasuriya KA, Johnson NW (1996) Sensitivity and specificity of OraScan ${ }^{\circledR}$ toluidine blue mouthrinse in the detection of oral cancer and precancer. J Oral Pathol Med 25:97-103

Warnakulasuriya S, Johnson NW, Van der Waal I (2007) Nomenclature and classification of potentially malignant disorders of the oral mucosa. J Oral Pathol Med 36(10):575-580

Wen-You L, Jin-Gou X, Xi-Wen H (2000) Characterization of the binding of methylene blue to DNA by spectroscopic. Methods Anal Letters 33(12):2453-2464

Wilson EB (1927) Probable inference, the law of succession, and statistical inference. J Am Stat Assoc 22:209-212

Ya-Wei C (2007a) Application of in vivo stain of methylene blue as a diagnostic aid in the early detection and screening of oral squamous cell carcinoma and precancerous lesion. J Chin Med Assoc 70:497-503 
Ya-Wei C (2007b) Methylene blue as a diagnostic aid in the early detection of oral cancer and precancerous lesion. Br J Oral Maxillofac Surg 45:590-591

Yu DS, Chang SY, Ma CP (1990) Photoinactivation of bladder tumor cells by methylene blue: study of a variety of tumor and normal cells. J Urol 144(1):164-168

doi:10.1186/2193-1801-2-95

Cite this article as: Riaz et al:: Methylene blue as an early diagnostic marker for oral precancer and cancer. SpringerPlus 2013 2:95.

Submit your manuscript to a SpringerOpen ${ }^{\circ}$ journal and benefit from:

- Convenient online submission

- Rigorous peer review

- Immediate publication on acceptance

- Open access: articles freely available online

- High visibility within the field

- Retaining the copyright to your article

Submit your next manuscript at $\boldsymbol{\nabla}$ springeropen.com 